\title{
Multi-Beam Uncoordinated Random Access MAC for Underwater Communication Networks
}

\author{
Bryan Ehlers \\ University of Iowa \\ Iowa City, IA, USA \\ bryan-ehlers@uiowa.edu
}

\author{
Ananya Sen Gupta \\ University of Iowa \\ Iowa City, IA, USA \\ ananya-sengupta@uiowa.edu
}

\author{
Ryan McCarthy \\ University of Iowa \\ Iowa City, IA, USA \\ ryan-mccarthy-1@uiowa.edu
}

\begin{abstract}
We consider a multi-beam directional network where the nodes have digital antenna arrays capable of performing post-reception digital beamforming without prior knowledge of angle-of-arrival. Post-reception digital beamforming has greatly advanced uncoordinated random access in directional networks where, in the past, scheduling of beams was a bottleneck. A key trade off in these systems is number of antennas and corresponding beam-width We demonstrate, through numerical simulations, that the random access MAC under consideration, MB-URAM, does not perform as well as the idealized version originally presented [Kuperman et al. 2016]. We demonstrate how interference between coincident nodes cause a significant number of packet drops even with a very large number of antennas. Finally, we suggest how to mitigate the effect of the interference and introduce ideas to do so.
\end{abstract}

\section{KEYWORDS}

Medium access control, MAC, random access, multibeam, beamforming, directional networking

\section{ACM Reference format:}

Bryan Ehlers, Ananya Sen Gupta, and Ryan McCarthy. 2018. Multi-Beam Uncoordinated Random Access MAC for Underwater Communication Networks. In Proceedings of The 13th ACM International Conference on Underwater Networks Systems, Shenzhen, China, December 3-5, 2018 (WUWNet'18), 6 pages.

https://doi.org/10.1145/3291940.3291979

\section{INTRODUCTION}

Digital beamforming has been widely used in multi-beam directional networks and has given rise to higher transmit rates and longer transmit ranges by providing power gain proportional to the number of receive antenna elements [Tse and Viswanath 2005]. Additionally, digital beamforming can permit receive beams to be formed to a particular transmitter without the receiver knowing where the transmitter is located [Litva and Lo 1996] [Veen and Buckley 1988]. A random access radio frequency (RF) medium access controller (MAC) for such a directional multiple beam network

Permission to make digital or hard copies of all or part of this work for personal or classroom use is granted without fee provided that copies are not made or distributed for profit or commercial advantage and that copies bear this notice and the full citation on the first page. Copyrights for components of this work owned by others than ACM must be honored. Abstracting with credit is permitted. To copy otherwise, or republish, to post on servers or to redistribute to lists, requires prior specific permission and/or a fee. Request permissions from permissions@acm.org.

WUWNet'18, December 3-5, 2018, Shenzhen, China

(C) 2018 Association for Computing Machinery.

ACM ISBN 978-1-4503-6193-4/18/12 ..\$15.00

https://doi.org/10.1145/3291940.3291979 has been proposed in [Kuperman et al. 2016]. This work characterizes the application of the RF system in [Kuperman et al. 2016] to enable an acoustic communication network of underwater nodes. In addition, we propose and an extension to make the original technique in [Kuperman et al. 2016] operational across more scenarios including small clusters of beam-coincident nodes as well as dense node environments.

In traditional underwater acoustic communication via a node with multiple transducer elements, a beam would be formed by introducing phase shifts to each of the individual transducers, so that the combination forms a beam in the appropriate direction. In such a system, beams must be preplanned and prescheduled so that transmit and receive beams are pointing in the correct direction for successful transmission and reception (link closure) to occur. This type of scheduled MAC has been investigated in prior work under the assumption that all node transmissions and receptions are synchronized [Zhang 2005]. The need for nodes to be synchronized is prohibitive in scenarios where nodes do not have a shared clock or a reliable GPS reference.

In modern digital beamforming systems, the samples of the received signal taken at each receive element of the array can be processed in an adaptive fashion allowing for beams to be formed without prior knowledge of the transmitter location [Sayers et al. 2012]. This type of adaptive beamforming processing enables uncoordinated MAC policies. If the receive beams can be formed without prior knowledge of the other users' locations a predetermined schedule is not required for a listening node to successfully received the signal from a transmitting node. In this work, we assess an uncoordinated MAC policy for a digital beamforming system, as proposed in [Kuperman et al. 2016], and suggest an improvement to the scheme.

\section{TECHNICAL APPROACH}

\subsection{System model}

We consider a network consisting of $N$ nodes, whose locations are uniformly distributed according to a two-dimensional spatial Poisson process with an average of $N$ nodes per $\pi r^{2}$ area, where $r$ is the radius of the circular simulation environment. This is illustrated in Figure 1. This work assumes that all nodes can either transmit or receive at a given point in time, ie. half-duplex. We do not allow a single node to transmit and receive at the same time.

Before characterization of the two proposed techniques, we wish to determine a theoretical upper bound on total network throughput to help us understand the maximum possible throughput of any given network of $N$ nodes regardless of real world effects such as interference or insufficient received signal strength. 


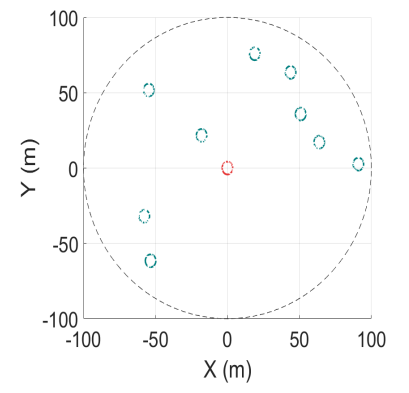

Figure 1: Simulation layout of nodes uniformly distributed throughout a circle according to a two dimensional spatial Poisson process of radius $\mathbf{1 0 0}$ meters. We consider centrally located red node when computing average node throughput.

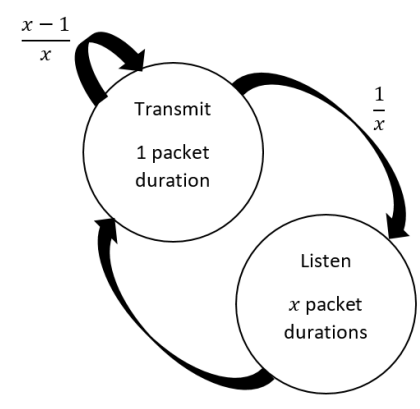

Figure 2: State machine for a node operating under the MB-URAM policy. The nodes are uncoordinated so that each node transitions between states independently of other nodes. That is, each node is operating on its own clock.

Consider a network consisting of $N$ nodes, where each node may form as many links as they need. If we let $N_{T X}$ of the nodes be transmitters then we have $\left(N-N_{T X}\right)$ remaining nodes to be receivers. Since each of the $N_{T X}$ transmitters could form links to each of the receivers, there would be a total of $N_{T X}\left(N-N_{T X}\right)$ active links. Assuming that a packet takes one unit of time to transmit, if all of the links facilitate a single successful packet transmission, the total network throughput in packets/unit time is equal to the number of possible links, $N_{T X}\left(N-N_{T X}\right)$ per that unit of time occupied by a single packet transmission. It follows then that the maximum network throughput is found by maximizing $N_{T X}(N-$ $\left.N_{T X}\right)$ with respect to $n$. The objective function $\phi\left(N_{T X}\right)=N_{T X}(N-$ $N_{T X}$ ) has a derivative $\frac{d \phi}{d N_{T X}}=N-2 N_{T X}$. Setting $\frac{d \phi}{d N_{T X}}$ equal to zero, the maximizing $N_{T X}^{*}=\frac{N}{2}$. That is, to achieve a maximum possible total network throughput, half of the nodes should be transmitting and half of the nodes should be receiving. At any given time, there are $\frac{N}{2} * \frac{N}{2}$ potentially active links in the network. If all links supported a successful transmission of one packet per unit time then the maximum network throughput in packets per unit time is given by the number of active links, $\phi\left(N_{T X}^{*}\right)=\frac{1}{4} N^{2}$ [Kuperman et al. 2016].

\subsection{Multi-Beam Uncoordinated Random Access MAC (MB-URAM) policy}

A multi-beam network is one in which each node is equipped with an $M$-element uniform linear array with half wavelength spaced elements. Post-reception adaptive beamforming allows each node to form up to $M$ simultaneous orthogonal beams [Litva and Lo 1996]. The information being transmitted on each beam may be independent. Thus, each receiving node is capable of simultaneously closing links with a maximum of $M$ transmitting nodes where each transmitting node is located in a different non-overlapping beam to keep the signals from interfering with one another. In the event two transmitting nodes are located within a single receive beam, the system of [Kuperman et al. 2016] will not successfully close the link with either transmitter.

From the previous work of Kuperman et. al. [Kuperman et al. 2016], it is shown that MB-URAM, for the special (albeit nonrealistic) case of sufficient received signal power for every attempted link, achieves the maximum network throughput for a multi-beam network for a specific type of packet traffic that we describe next. The policy each MB-URAM node uses to govern its packet transmitting behavior is as follows: if a node has a packet to send, it transmits to a maximum of $M$ in $M$ different non-interfering transmit beams nodes, and then goes to a listening state for a random number of packet durations where it forms up to $M$ beams to attempt to receive. More specifically, Figure 2 shows the operation of a typical node using the MB-URAM policy. After a node is done transmitting, either it will choose to transmit again with probability $\frac{x-1}{x}$ or it will go to a listening state for $x$ packet durations with probability $\frac{1}{x}$. When a node is in transmit state, it will remain for one packet duration and when in listening state, it will remain for $x$ packet durations. This behavior is summarized pictorially in Figure 2.

The parameter $x$ governs the packet transmission behavior of a node. Figure 3 shows the activity of a typical node operating under the MB-URAM policy. From this, it is clear that varying the parameter $x$ controls how often a node transmits packets to the channel and how long a node is in listening state. It is shown in [Kuperman et al. 2016] that for the case of sufficient received power for all potential links, the theoretical bound on network throughput can be reached with the MB-URAM system as $x$ approaches infinity, if a sufficient number of antennas are used, and for large $N$.

We have re-run the analysis in [Kuperman et al. 2016] but for a more realistic case in which transmitted signals are attenuated by the channel and are therefore received at a weaker signal level at distant nodes as compared to the signal level at closer nodes. The powers are such that two nodes can always close a link but for this case, the MB-URAM system does not achieve the theoretical upper bound, even in the limit as $\mathrm{x}$ goes to infinity. Moreover, we are more interested in moderate (thus, realistic) values of $x$. See Figure 4 shows the total network throughput as a function of the number of nodes for three different values of $x$.

In order to re-run the analysis in [Kuperman et al. 2016] with the realistic signal attenuation a metric must be used to decide when to drop packets. An interfering user being within a beam-width is no longer appropriate since some distant interfering user may not cause enough interference to spoil the link with a closer user. 


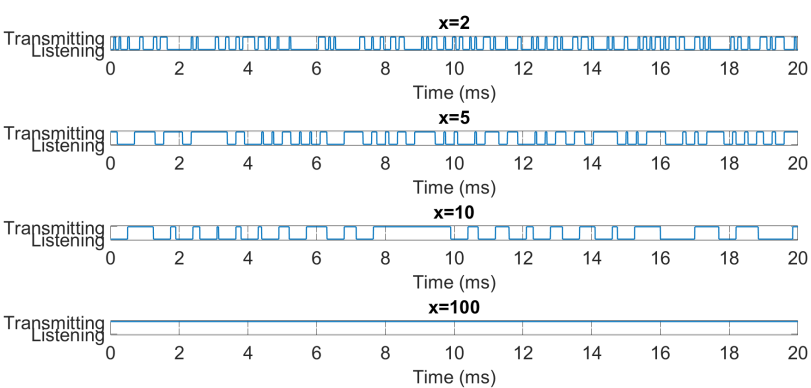

Figure 3: Node state time evolution for different values of the parameter $x$. The larger values of $x$ means that a node will transmit with higher probability and when a listening state is chosen, it will last longer. Likewise, for smaller values of $x$, a node will enter the listening state with higher probability and will remain listening for a shortened amount of time. Because of this, smaller values of $x$ results in many bursts where a node toggles more frequently between states.

Instead of the packet drop criteria from [Kuperman et al. 2016], we use SINR to help us determine the rate at which each user can communicate reliably. We opt to use a fixed rate of $1 \mathrm{bit} / \mathrm{channel}$ use:

$$
R_{\text {MAX }}=\frac{1}{2} \log _{2}(1+S I N R) \geq 1 \text {, bits / channel use }
$$

The simulation was setup as follows: the nodes are uniformly distributed according to a two-dimensional spatial Poisson process with an average of $N$ users per $\pi r^{2}$ circular area, nodes all have the same transmit power, packet duration time and idle duration time block lengths, but each has its own randomly chosen start time so that packets collide asynchronously at any given receiver. This is consistent with the previous work [Kuperman et al. 2016].

\section{APPLICABILITY TO UNDERWATER NETWORKS}

The computational techniques developed in this work are broadly applicable to underwater wireless sensor networks where interference between nodes is a practical reality. This work is particularly attractive to environmental monitoring applications that deploy underwater sensor networks where each node is equipped with two or more transducer elements and is capable of adaptive beamforming at each receiving node.

Our theoretical setup and analysis assumes close-range wireless transmission without significant channel delay spread between two nodes within the underwater network. For example, this work will be relevant to assessment and mitigation of inter-node interference within a network of underwater sensors where the direct arrival path between any two sensors significantly dominates over any multipath arrivals. As such, the proposed technique is applicable to freshwater closely spaced radio-frequency $(\mathrm{RF})$ networks [Teixeira et al. 2014], but not so much within salt-water RF paradigm due to high conductivity of the medium [Shaw et al. 2006]. Our method

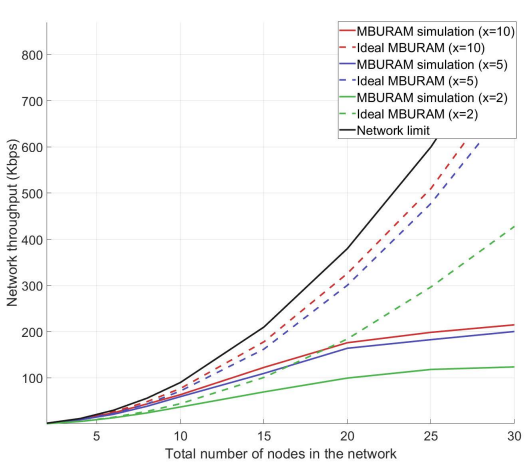

Figure 4: Throughput performance of MB-URAM with 20 symbol packets and 50 antennas. Despite the large number of antennas, it can be seen that due to the large number of nodes, the interference hinders the performance significantly below that of the ideal policy studied in [Kuperman et al. 2016] and even further below that of the absolute limit of a multi-beam network. The dashed lines represent ideal (unrealistic) received power, solid lines represent realistic power attenuation, and the solid black line is the limit for a multi-beam network regardless of policy.

is also applicable to underwater acoustic networks with medium to long-range node spacing, where multipath scattering does not dominate the channel delay spread.

We now provide shallow water acoustic channel simulations using [Qarabaqi and Stojanovic 2013] for diverse oceanic conditions over medium and long ranges and discuss in each case the applicability of our technique to underwater acoustic networks. Specifically, we consider three scenarios in Figures 1-3: (i) shallow water depth (20 meters) medium-range (200 meters), (ii) shallow water depth (20 meters) long-range (1000 meters), and (iii) mediumwater depth (60 meters) long-range (1000 meters). In each case, the transmitter and receiver were kept 10 meters from the ocean surface to examine the effect of multipath arrivals between the transmitter and receiver.

Figure 5 illustrates a channel simulation where the multipath interference is strong due to shortness of range (200 meters) and depth (20 meters), and therefore, not the intended paradigm for successful application of our method. On the other hand, multipath interference is drastically less in the simulated channel in Figure 6, which has same water depth but significantly longer range (1000 meters), which presents a well-suited case for successful application of our method. Finally, Figure 7 illustrates a simulated channel with greater water depth (60 meters) and long range (1000 meters) where the multipath arrivals are reduced in energy and manifest farther from the direct arrival in the channel delay spread. Our method may be directly applied to this channel after implementing an additional layer of real-time channel equalization.

The shallow water acoustic channel impulse response is closely shaped by the acoustic delay spread due to scattering of the transmitted acoustic energy by the ocean and reflections from the moving sea surface and static sea bottom. The lower-energy but persistent 
components of the channel delay spread, typical for shallow water medium ranges, are attributed mainly to environmental scattering, such as due to diffused reflections from the static sea bottom. The high-energy rapid channel fluctuations, which may pose significant interference to the direct arrival, are primarily due to specular reflections and focusing events [Preisig and Deane 2004] by the moving sea surface. As the range and water depth increases, greater acoustic scattering as well as increased absorption by multiple reflections results in less interference from multipath arrivals.

It is outside the scope of this work to provide specific channel estimation or equalization schemes, which are well-documented in the undersea signal processing and acoustic communications literature (refer e.g. [Gupta and Preisig 2012; Stojanovic and Preisig 2009; Stojanovic et al. 1996] and references therein). Based on our discussion across case studies above, we will assume that the underwater network is deployed over a combination of water depths and ranges (e.g. Figures 6 and 7) where the interference from fluctuating multipath is either minimal or structured enough to be robustly compensated using popular equalization techniques [Stojanovic et al. 1996].

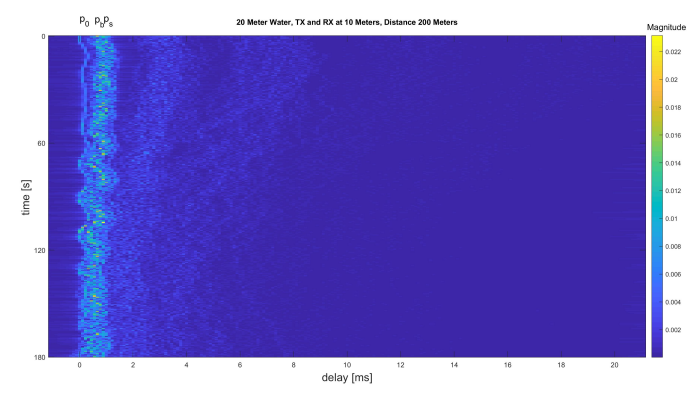

Figure 5: Simulated underwater acoustic channel with 20meter water depth. The transmitter and receiver are set at 10-meters high at 200-meters apart. This simulates a shallow water scenario with significant interference due to reflections from the ocean surface or sea bottom. It is difficult to recover the direct-path signal in this situation because there are less reflections and thus the absorption of the signal is much less causing larger magnitude delay spread in the node-to-node channel.

\section{RESULTS}

The post-beamformed matched filter SINR at each receiving node can be used to determine if the minimum rate can be achieved or not. If the SINR falls below the threshold for any of the symbols in a packet, the packet is dropped. Thus, if $R_{M F}^{M A X}<1$ bits/channel use then the matched filter receiver drops the packet. In other words, required to successfully receive 1 bits/channel use the SINR for any of the symbols during the packet must not fall below the threshold, or else the packet is dropped.

For the simulation, we use 20 BPSK symbols per packet and operate at $4 \mathrm{Kbps}$. For the various plots produced, it is important to realize that the matched filter is being performed after the beamgain is applied. The SNR is the signal to noise ratio without any

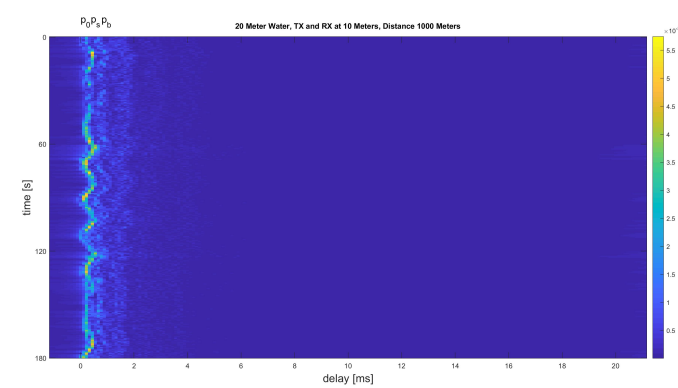

Figure 6: Simulated underwater acoustic channel with 20meter water depth. The transmitter and receiver are set at 10meters high at 1000-meters apart. This simulates a shallow water scenario with low multipath interference that is most compatible with our method, which is designed to mitigate node-to-node interference rather than multipath interference. Because of the shallow water depth and long-range distance between the transmitter and receiver, it takes longer and more reflections, and hence more energy absorption, for the multipath arrivals to reach the receiver.

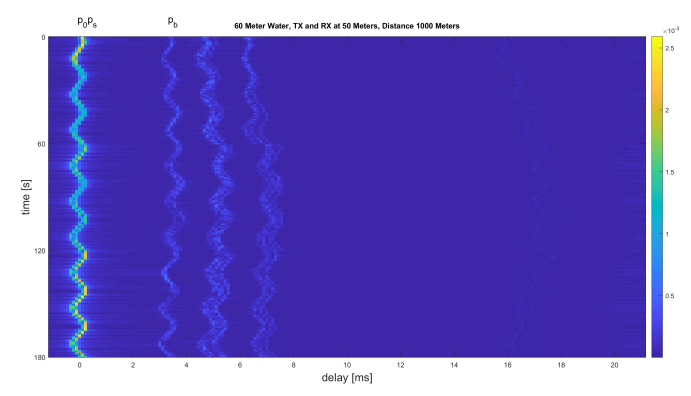

Figure 7: Simulated underwater acoustic channel with 60meter water depth. The transmitter and receiver are set at 50-meters high at 1000-meters apart. This simulates a deepwater scenario with very weak and separable interference due to reflections from the surface or sea bottom. The delayed multipath arrivals can be seen more clearly in this scenario making it easier to recover the signal.

interference present. We simulate for a $S N R_{\min }=5 \mathrm{~dB}$, where $S N R_{\text {min }}$ is the SNR of the weakest user (farthest user from the centrally located receiver) and nodes that are closer will benefit from a higher SNR.

In Figure 8, it can be seen that utilizing more antennas we are able to mitigate the effect of the interference and achieve a higher throughput for centrally located node, see Figure 1. Adding more antennas is decreasing the beamwidth approximately according to $\frac{100^{\circ}}{M}$ [Litva and Lo 1996] which gives the central receiving node a higher SINR. A wider beamwidth introduces more interference as the beam will contain many nodes especially when the number of nodes in the network grows large. Note that in Figure 8 we see that adding more antennas allows the throughput to remain high as 


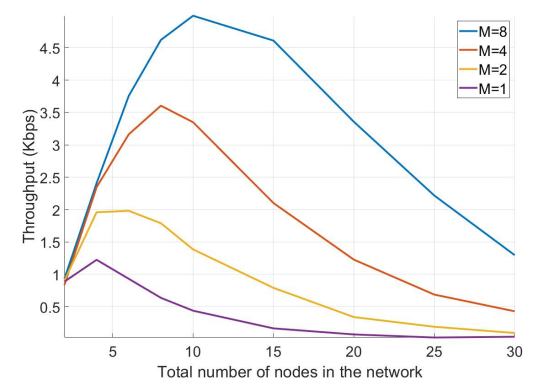

Figure 8: Effect of varying the number of antennas elements each node has, central nodes's average throughput achieved for $S N R_{\text {min }}=5, \mathbf{x}=10$, packet size of 20 symbols, and bit rate of $4 \mathrm{Kbps}$. The network area is $\pi 100^{2}$ square meters.

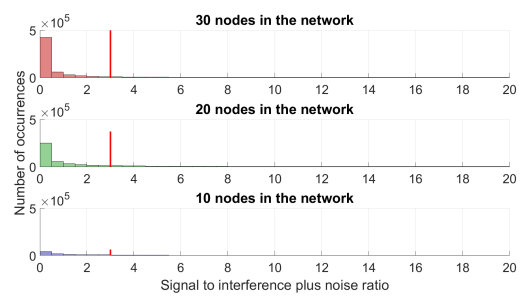

Figure 9: Histogram of the SINR for when links fail using 8 antennas. The result was obtained by running 100 simulations of 1000 timeslots each. The bold red line indicates the cut-off SINR necessary to achieve the desired communication rate. As nodes are added to the network, the number of occurrences of insufficient SINR increases faster than any other group of bins. This shows that significant interference is causing dropped packets when the node density is large.

more nodes are added, but eventually, the interference experienced is too much for the beamformer alone to mitigate.

From Figure 9 we can see that the number of times a node sees an SINR below the threshold required (which causes a dropped packet) grows as more nodes are added to the network. This is due to more nodes being present in the same direction as the node attempting to transmit. For example, a node may be attempting to transmit a packet to the central node but another node is angle coincident and closer than the node of interest. The packet transmission of interest will most likely be corrupted by the large interference signal and the packet will be dropped if the SINR threshold is not achieved.

Interference mitigation techniques can be utilized to help with this problem. Many different multiuser detectors (MUD) have been explored for wireless systems [Verdu 1998] and have potential to further increase the throughput of such a directional beamforming network. A receiver can use MUD in an intelligent way as well. For example, if the traditional receiver alone drops the packet, there must have been interference coming from the direction of the beam that is trying to form a link. In these situations, one could try using the MUD processing and attempt to receive the packet.

\section{CONCLUSION}

In this paper, we reviewed a Multi-Beam Uncoordinated Random Access MAC (MB-URAM) for digital beamforming systems. We have shown that when realistic constraints are set such as a fixed transmit power and minimum acceptable communicating rate, interference limits the performance to well below that of the ideal scenario studied in [Kuperman et al. 2016]. Even for very large arrays (50 antenna elements) the performance is not able to approach the upper limit. It was shown through numerical simulations that for a fixed transmit power and communication rate, packet drops become prohibitive in dense node environments when more realistic number of antenna elements are unable to null out interference. Future directions of research include more sophisticated receiver designs and different network topology.

\section{ACKNOWLEDGEMENT}

This work is partially funded by Air Force Contract No. FA872105-C-0002 and/or FA8702-15-D-0001, and Office of Naval Research ocean acoustics program (grant no. N00014-18-1-2081). Any opinions, findings and conclusions or recommendations expressed in this material are those of the authors and do not necessarily reflect the views of the United States Government. We also thank Dr. Rachel Learned of MIT Lincoln Laboratory for her comments.

\section{REFERENCES}

Ananya Sen Gupta and James Preisig. 2012. A geometric mixed norm approach to shallow water acoustic channel estimation and tracking. Physical Communication 5, 2 (2012), 119-128.

G. Kuperman, R. Margolies, N. M. Jones, B. Proulx, and A. Narula-Tam. 2016. Uncoordinated MAC for Adaptive Multi-Beam Directional Networks: Analysis and Evaluation. In 2016 25th International Conference on Computer Communication and Networks (ICCCN). 1-10. https://doi.org/10.1109/ICCCN.2016.7568593

John Litva and Titus K. Lo. 1996. Digital Beamforming in Wireless Communications (1st ed.). Artech House, Inc., Norwood, MA, USA.

James C Preisig and Grant B Deane. 2004. Surface wave focusing and acoustic communications in the surf zone. The fournal of the Acoustical Society of America 116, 4 (2004), 2067-2080.

Parastoo Qarabaqi and Milica Stojanovic. 2013. Statistical characterization and computationally efficient modeling of a class of underwater acoustic communication channels. IEEE Journal of Oceanic Engineering 38, 4 (2013), 701-717.

A. E. Sayers, W. M. Dorsey, K. W. O'Haver, and J. A. Valenzi. 2012. Planar Near-Field Measurement of Digital Phased Arrays Using Near-Field Scan Plane Reconstruction. IEEE Transactions on Antennas and Propagation 60, 6 (June 2012), 2711-2718. https: //doi.org/10.1109/TAP.2012.2194666

Andrew Shaw, AI Al-Shamma'a, SR Wylie, and D Toal. 2006. Experimental investigations of electromagnetic wave propagation in seawater. In 2006 European Microwave Conference. IEEE, 572-575.

Milica Stojanovic and James Preisig. 2009. Underwater acoustic communication channels: Propagation models and statistical characterization. IEEE communications magazine 47, 1 (2009), 84-89.

M Stojanovic, JG Proakis, and JA Catipovic. 1996. Performance of high-rate adaptive equalization on a shallow water acoustic channel. The fournal of the Acoustical Society of America 100, 4 (1996), 2213-2219.

Filipe Teixeira, Pedro Freitas, Luís Pessoa, Rui Campos, and Manuel Ricardo. 2014. Evaluation of IEEE 802.11 Underwater Networks Operating at $700 \mathrm{MHz}, 2.4 \mathrm{GHz}$ and $5 \mathrm{GHz}$. In Proceedings of the International Conference on Underwater Networks \& Systems. ACM, 11.

David Tse and Pramod Viswanath. 2005. Fundamentals of Wireless Communication. Cambridge University Press, New York, NY, USA.

B. D. Van Veen and K. M. Buckley. 1988. Beamforming: a versatile approach to spatial filtering. IEEE ASSP Magazine 5, 2 (April 1988), 4-24. https://doi.org/10.1109/53.665

Sergio Verdu. 1998. Multiuser Detection (1st ed.). Cambridge University Press, New York, NY, USA.

Zhensheng Zhang. 2005. Pure directional transmission and reception algorithms in wireless ad hoc networks with directional antennas. In IEEE International Conference on Communications, 2005. ICC 2005. 2005, Vol. 5. 3386-3390 Vol. 5. https://doi.org/ 10.1109/ICC.2005.1495049 